\title{
Screening potential and continuum lowering in a dense plasma under solar-interior conditions ${ }^{\star}$
}

\author{
Jiaolong Zeng ${ }^{1}$, Yongjun $\mathrm{Li}^{3}$, Cheng $\mathrm{Gao}^{3}$, and Jianmin $\mathrm{Yuan}^{2,3}$ \\ ${ }^{1}$ College of Science, Zhejiang University of Technology, Hangzhou, Zhejiang 310023, PR China \\ e-mail: jiaolongzeng@hotmail.com \\ 2 Graduate School of China Academy of Engineering Physics, Beijing 100193, PR China \\ e-mail: jmyuan@nudt.edu.cn \\ 3 Department of Physics, College of Liberal Arts and Sciences, National University of Defense Technology, Changsha, \\ Hunan 410073, PR China
}

Received 3 December 2019 / Accepted 9 January 2020

\begin{abstract}
An accurate description of the screening potential induced by a hot, dense plasma is a fundamental problem in atomic physics and plasma physics, and it plays a pivotal role in the investigation of microscopic atomic processes and the determination of macroscopic physical properties, such as opacities and equations of state as well as nuclear fusion cross sections. Recent experimental studies show that currently available analytical models of plasma screening have difficulty in accurately describing the ionization-potential depression, which is directly determined by the screening potential. Here, we propose a consistent approach to determine the screening potential in dense plasmas under solar-interior conditions from the free-electron micro-space distribution. It is assumed that the screening potential for an ion embedded in a dense plasma is predominately determined by the free electrons in the plasma. The freeelectron density is obtained by solving the ionization-equilibrium equation for an average-atom model to obtain the average degree of ionization of the plasma. The proposed model was validated by comparing the theoretically predicted ionization-potential depression of a solid-density Si plasma with recent experiments. Our approach was applied to investigate the screening potential and ionizationpotential depression of $\mathrm{Si}$ plasmas under solar-interior conditions over a temperature range of $150-500 \mathrm{eV}$ and an electron-density range of $5.88 \times 10^{22}-3.25 \times 10^{24} \mathrm{~cm}^{-3}$. It can be easily incorporated into atomic-structure codes and used to investigate basic atomic processes, such as photoionization, electron-ion collisional excitation and ionization, and Auger decay, in a dense plasma.
\end{abstract}

Key words. atomic data - atomic processes - Sun: interior - stars: interiors - dense matter

\section{Introduction}

The Sun is a prototypical star that can be used for investigating stellar processes and for testing theories of stellar structure and evolution. In the solar interior, the plasma is dense, and stellar processes are thus significantly influenced by plasma screening. Although the solar interior is not directly accessible to observations, plasma screening shows its effects in astrophysical phenomena, such as solar oscillations (helioseismology) (Antia 2009) because it affects the opacity and equation of state. The screening potential induced by the plasma environment plays pivotal roles in both microscopic atomic processes and macroscopic physical properties (Basu \& Antia 2008; Bahcall et al. 2004). Microscopically, it affects the atomic structure and all kinds of radiative and collisional atomic processes, such as photo-excitation and photoionization, as well as electron-impact excitation and the ionization of ions (Vinko et al. 2015; van den Berg et al. 2018). Macroscopically, the ionization balance, and hence, the physical properties of equations of state, opacities, and thermal and electronic conductivities can be significantly modified by the dense plasma environment (Bailey et al. 2015; Rogers \& Iglesias

* Tables of the numerical data used for the figures are only available at the CDS via anonymous ftp to cdsarc.u-strasbg.fr (130.79.128.5) or via http://cdsarc.u-strasbg.fr/viz-bin/ cat/J/A+A/634/A117
1994; Iglesias \& Rogers 1991; Rose 1992; Hansen et al. 2005; Vinko et al. 2012). Moreover, in the dense plasma, the nuclear fusion cross section and rate can be significantly enhanced over the binary Gamow (1928) results because of the plasma screening effect (Yakovlev \& Shalybkov 1989; Ichimaru 1993); therefore, they can affect the stellar evolution.

One key physical quantity is continuum lowering, which is also referred to as ionization-potential depression (IPD) and is directly determined by the plasma screening potential. Recent technological advances have made it possible to measure the IPD of dense plasmas directly (Ciricosta et al. 2012, 2016; Hoarty et al. 2013; Fletcher et al. 2014). However, the predictions of widely used analytical models (Stewart \& Pyatt 1966; Ecker \& Kröll 1963; Rozsnyai 1972; Debye \& Hückel 1923) disagree with the experimental measurements. The first experiment (Ciricosta et al. 2012), which was performed at the Linac Coherent Light Source (LCLS), shows that the Ecker-Kröll model (Ecker \& Kröll 1963) is in much better agreement with the observations than the Stewart-Pyatt model (Stewart \& Pyatt 1966). However, laser-driven shock experiments at the ORION laser system are in better agreement with the Stewart-Pyatt model (Stewart \& Pyatt 1966) than the Ecker-Kröll model (Ecker \& Kröll 1963). Moreover, a later experiment at LCLS shows that the predictions of the Ecker-Kröll model for the IPD of $\mathrm{Mg}$ and $\mathrm{Al}$ compounds disagree with the experimental results (Ciricosta et al. 2016). Comparisons of theoretical predictions 
with recent experiments have further revealed that the analytical models (Stewart \& Pyatt 1966; Ecker \& Kröll 1963; Rozsnyai 1972; Debye \& Hückel 1923) have difficulty in capturing the essential features of hot, dense plasmas at solid and abovesolid densities. This lack of a consistent theoretical formulation for the IPD of hot dense plasmas has stimulated a variety of theoretical investigations (Preston et al. 2013; Iglesias 2014; Hansen et al. 2014; Crowley 2014; Son et al. 2014; Calisti et al. 2015; Vinko et al. 2014; Stransky 2016; Lin et al. 2017; Rosmej 2018; Kasim et al. 2018; Ali et al. 2018; Hu 2017; Röpke et al. 2019; Kraus et al. 2018).

In the solar interior, the plasma is dense, and has an electron density above $1.0 \times 10^{22} \mathrm{~cm}^{-3}$, and thus the plasma screening potential strongly influences the ionization balance and atomic processes. Physical properties, such as the opacities and equations of state, play important roles in the determination of the internal solar structure. However, the current standard solar model (Bahcall \& Ulrich 1988; Guenther et al. 1992) disagrees with helioseismic studies (Bahcall et al. 2004; Basu \& Antia 2008) that employ the most updated Rosseland mean opacity for a solar mixture. This open problem of the "missing opacity" in the solar interior can be resolved if we increase the opacity. Accurate calculations of the radiative opacity require a consistent description of the plasma screening potential. However, atomic data that take the density effect into account are extremely lacking for dense plasmas under solar-interior conditions. Very recently, Deprince et al. have investigated plasma-environment effects on the $\mathrm{K}$ lines of oxygen (Deprince et al. 2019a) and iron (Deprince et al. 2019b) ions in accretion disks around black holes. Magnetohydrodynamic simulations show that the electron temperatures and densities span the ranges of $10^{5}-10^{7} \mathrm{~K}$ and $10^{18}-10^{22} \mathrm{~cm}^{-3}$, respectively (Schnittman et al. 2013). The electron density in the accretion disk of a black hole is much lower than that in the solar interior, and Deprince et al. used the Debye-Hückel model to describe the plasma-screening effect. Such an analytical model is not valid for solar- and stellar-interior plasmas, as mentioned above. There is thus an urgent need to improve the treatment of density effects in higher density plasmas.

In this work, we aim to develop a consistent theoretical formulation of the plasma screening potential for the dense plasmas under solar-interior conditions. The screening potential can be further incorporated into an atomic-structure code to investigate microscopic atomic processes, including the excitation and ionization caused by photons and electrons. As an application of our theory, we investigate the plasma screening potential and IPD of Si plasmas under solar-interior conditions. It is thought that the atomic processes that occur in the dense environment of the solar interior may show interesting and novel phenomena, which may help to resolve the current discrepancy between the internal solar structure predicted by the standard solar model (Bahcall \& Ulrich 1988; Guenther et al. 1992) and the constraints from helioseismic observations (Bahcall et al. 2004; Basu \& Antia 2008).

\section{Theoretical method}

For an isolated atom and ion, the wave functions and atomic structure are determined by solving quantum mechanical equations [see for example (Jönsson et al. 2007; Gu 2008)] based on the Hamiltonian for which atomic units are used unless otherwise specified

$H=\sum_{i=1}^{\mathrm{N}} H_{D}(i)+\sum_{i<j}^{\mathrm{N}} \frac{1}{r_{i j}}$ where $H_{D}(i)$ is the single-electron Dirac Hamiltonian for the potential due to the nuclear charge and $N$ denotes the number of electrons. The basis states of the atomic system are constructed from products of $N$ one-electron Dirac spinors,

$\varphi_{n \kappa m}=\frac{1}{r}\left(\begin{array}{c}P_{n \kappa}(r) \chi_{\kappa m}(\theta, \psi, \sigma) \\ i Q_{n \kappa}(r) \chi_{-\kappa m}(\theta, \psi, \sigma)\end{array}\right)$

where $P_{n \kappa}(r)$ and $Q_{n \kappa}(r)$ are the large and small components of the radial wave functions, respectively, and $\chi_{\kappa m}(\theta, \psi, \sigma)$ is a twocomponent spherical spinor. The indices $n, \kappa$, and $m$ denote the principal, relativistic angular, and magnetic quantum numbers of the orbital, respectively.

In the central-potential approximation, the large and small components of the radial wave function satisfy coupled Dirac equations in the standard Dirac-Fock-Slater method:

$$
\begin{aligned}
& \left(\frac{\mathrm{d}}{\mathrm{d} r}+\frac{\kappa}{r}\right) P_{n \kappa}=\alpha\left(\varepsilon_{n \kappa}-V(r)+\frac{2}{\alpha^{2}}\right) Q_{n \kappa} \\
& \left(\frac{\mathrm{d}}{\mathrm{d} r}-\frac{\kappa}{r}\right) Q_{n \kappa}=\alpha\left(-\varepsilon_{n \kappa}+V(r)\right) P_{n \kappa}
\end{aligned}
$$

where $\alpha$ denotes the fine-structure constant and $\varepsilon_{n \kappa}$ is the energy eigenvalue of the electron orbital. The local central potential $V(r)$ includes contributions from the nuclear charge and from electron-electron interactions (Jönsson et al. 2007; Gu 2008), which can be determined by self-consistent iteration.

In a dense plasma characterized by an electron temperature $T$ and density $n_{\mathrm{e}}$, an electron that is bound to an ion feels a screening potential induced by the plasma environment (Li et al. 2008),

$V_{\mathrm{scr}}(r)=-\frac{Z}{r}+4 \pi\left[\frac{1}{r} \int_{0}^{r} r_{1}+\int_{\mathrm{r}}^{R_{0}}\right] r_{1} \rho\left(r_{1}\right) \mathrm{d} r_{1}-\frac{3}{2}\left[\frac{3}{\pi} \rho(r)\right]^{1 / 3}$,

where $Z$ is the nuclear charge and the radius of the ion sphere is determined by the ion number density: $R_{0}=\left(\frac{3}{4 \pi n_{i}}\right)^{\frac{1}{3}}$. The last term in Eq. (4) represents the Slater exchange potential. For $r>$ $R_{0}$, the potential is taken to have the constant value as it reaches $r=R_{0}$ (Son et al. 2014). The free-electron charge density $\rho(r)$ obeys the Fermi-Dirac distribution

$\rho(r)=\frac{1}{\pi^{2}} \int_{k_{0}(r)}^{\infty} \frac{k^{2} \mathrm{~d} k}{e^{\sqrt{k^{2} c^{2}+c^{4}}-c^{2}-V_{\mathrm{scr}}(r)-\mu / T}+1}$,

where $c$ denotes the speed of light in vacuum, $k$ is the momentum of the electron, $k_{0}(r)=\frac{\sqrt{2 V_{\mathrm{scr}}(r) c^{2}+V_{\mathrm{scr}}(r)^{2}}}{c}$, and $\mu$ denotes the chemical potential of the plasma.

The chemical potential is determined by the condition of electrical neutrality:

$\int_{0}^{R_{0}} 4 \pi r^{2} \rho(r) \mathrm{d} r=Z_{\mathrm{f}}$

where $Z_{\mathrm{f}}$ denotes the average degree of ionization, which is determined by the ionization balance of the plasma. This is usually obtained by solving the Saha ionization-equilibrium equation (Gao et al. 2013) for plasmas in local thermal equilibrium. In this code, the effect of IPD is considered when solving the Saha equation. When the ionization potential depression is properly considered, as in this work, this code correctly predicts the hydrogen ionization fraction in the solar interior (Christensen-Dalsgaard \& Döppen 1992). Here, we used the average-atom model instead to determine the average 
degree of ionization and the chemical potential by predicting the average orbital properties and occupation numbers at a finite temperature (Yuan 2002). We used the finite-temperature exchange-correlation functionals of Dharma-Wardana and Taylor (Dharma-wardana \& Taylor 1981) to construct the selfconsistent potential of the average-atom.

Once the radial wave functions are obtained, the energy-level structure of an isolated and screened ion can be determined, and then the atomic processes initiated by photons and electrons can be investigated. In this work, we focus mainly on the IPD because this physical quantity has been satisfactorily measured for solid-density plasmas. In order to calculate the IPD, it is necessary to solve the Dirac equation both for the isolated and screened ions. New functions that incorporate the screening potential into the atomic-structure calculation have also been implemented based on the flexible atomic code ( $\mathrm{Gu} 2008)$.

\section{Results and discussion}

Plasma screening effects can be reflected in atomic processes, such as electron-ion collisional excitation and ionization, photoionization, and Auger decay. Both the reaction thresholds and the cross-sections can be affected. However, it is difficult to separate a particular pathway from the myriad transitions that occur in a dense plasma, and thus experimental investigations of these processes are challenging. However, it is possible to measure physical properties, such as the radiative opacity for a dense plasma as a whole (Bailey et al. 2015). Advances in $\mathrm{X}$-ray laser technology have now made it possible to measure the IPDs of dense plasmas quantitatively both for pure elements and for mixtures (Ciricosta et al. 2012, 2016; Fletcher et al. 2014). Ultra-intense X-ray radiation is used to heat solid-density matter to the hot-dense regime. By increasing the photon energy of the laser across the K-shell ionization threshold, the K-edge, and thus the continuum lowering, can be measured by observing the onset of fluorescence emitted from the ions. We thus utilized the IPD to check the validity of our proposed model for the plasma screening potential.

In Fig. 1, we compare our theoretical IPDs for a solid-density Si plasma with the experimental measurements of Ciricosta et al. (2016). In their experiment, the laser photon energy was varied over the range 1290-1985 eV to open the K-edges of the ions. By observing the onset of the $K_{\alpha}$ fluorescence, they were able to measure the IPD for increasing charge states by increasing the photon energy. They determined the electron temperature and density of the plasma by solving the time-dependent rate equations that take into account the processes involved in the interaction between the X-rays and the Si plasma; detailed information can be found in their work (Ciricosta et al. 2016). They also give the theoretical results obtained from the analytical models of Ecker \& Kröll (Ecker \& Kröll 1963), the ion sphere (Rozsnyai 1972), and Stewart \& Pyatt (Stewart \& Pyatt 1966) in order to evaluate their predictive capabilities in dense plasmas. It is well known that the Debye-Hückel model (Debye \& Hückel 1923 ) is only valid for plasmas of lower densities, and thus they are not given here.

By inspecting Fig. 1, we find that our predictive IPDs are in good agreement with the experimental values for the lower ionization stages of $\mathrm{Si}^{4+}-\mathrm{Si}^{6+}$, although our theoretical result is somewhat smaller than the experimental value for $\mathrm{Si}^{7+}$. There are several possible reasons for the discrepancy between theory and experiment for the higher ionization stages. First, the higher charge states are produced at a later time during the interaction with the X-ray laser, and the energy of the photons is only

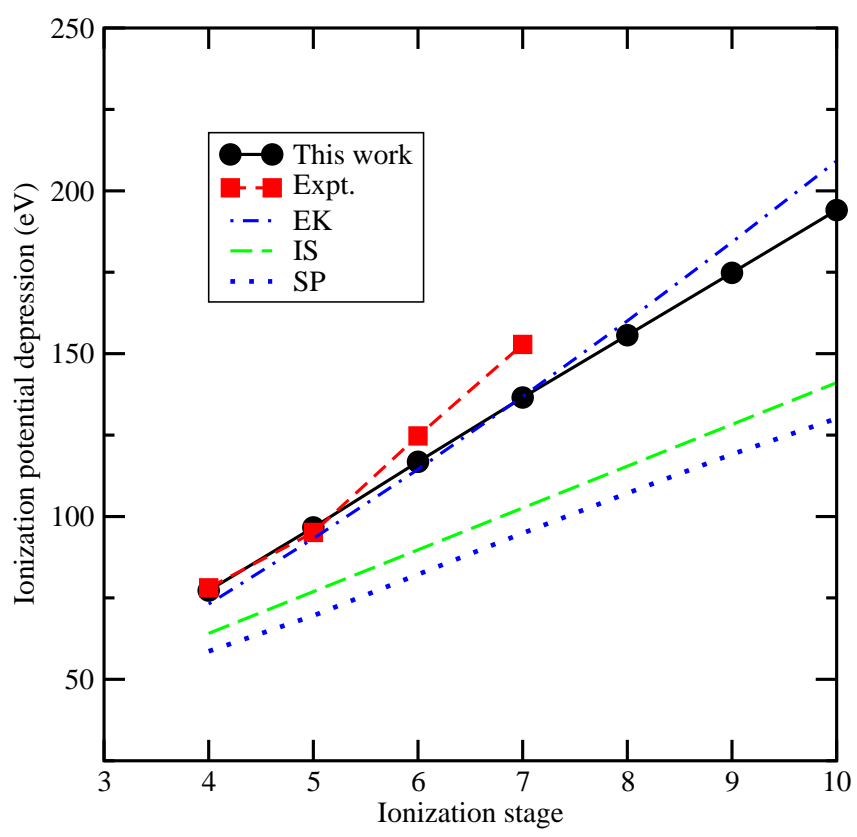

Fig. 1. Comparison of IPDs for solid-density Si plasmas predicted by our model with the experimental results (Expt) (Ciricosta et al. 2016). Theoretical results from the analytical models of Ecker \& Kröll (EK) (Ecker \& Kröll 1963), the ion-sphere model (IS) (Rozsnyai 1972), and Stewart \& Pyatt (SP) (Stewart \& Pyatt 1966) are given as well in order to evaluate their validity. The electron temperatures and densities are taken from a simulation (Ciricosta et al. 2016).

slightly above the ionization threshold of $\mathrm{Si}^{7+}$. Thus the energies of the free electrons around $\mathrm{Si}^{7+}$ that are produced by the $\mathrm{X}$-ray laser are very low. The highly ionized ion of $\mathrm{Si}^{7+}$ is thus surrounded by relatively "colder" free electrons, which changes the screening from the plasma. Second, the free electrons are not uniformly distributed in space; more free electrons follow the more highly ionized ions in order to maintain electrical neutrality. Finally, the plasma produced by the X-ray laser deviates considerably from thermal equilibrium, which also influences the screening.

Among the analytical models, the one of Ecker-Kröll (Ecker \& Kröll 1963) seems to be in much better agreement with the measurements than the ion-sphere (Rozsnyai 1972) and Stewart-Pyatt (Stewart \& Pyatt 1966) models. But it is known that the Ecker-Kröll model overestimates the IPD for the higher ionization stages (Hoarty et al. 2013), as in the present case. For dense plasmas, the analytical ion-sphere (Rozsnyai 1972) and Stewart-Pyatt (Stewart \& Pyatt 1966) models have been widely used in various fields to compute the basic atomic processes and physical properties of dense plasmas. They have been extensively applied to investigate atomic structure (Janev et al. 2016), photoionization (Qi et al. 2009), electron-impact excitation and ionization (Zhang et al. 2010; Li et al. 2017), and momentumtransfer cross-sections (Khrapak et al. 2003). Experimental elastic X-ray scattering suggests that plasma screening is important for the determination of the dense-plasma static structure factor (Saiz et al. 2008). The analytical models have also been used to calculate opacities and equations of state (Bailey et al. 2015; Rogers \& Iglesias 1994; Iglesias \& Rogers 1991; Rose 1992; Hansen et al. 2005; Vinko et al. 2012; Porcherot et al. 2011; Blancard et al. 2011; Zeng \& Yuan 2006, 2007; Gao et al. 2011). Moreover, the IPDs are used to diagnose plasma conditions, such as the density or temperature. Therefore an accurate description 


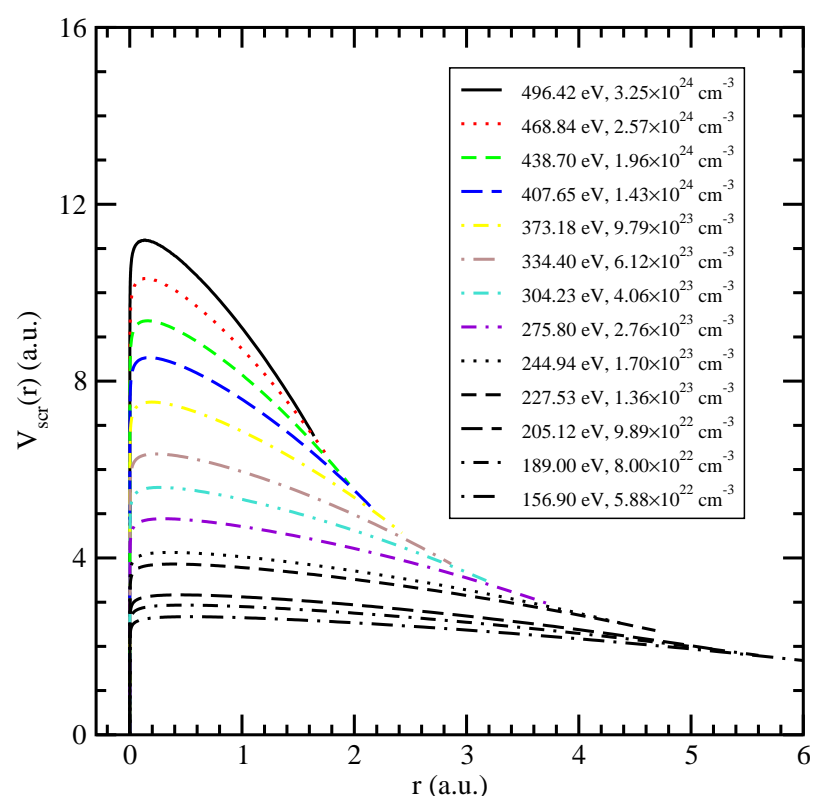

Fig. 2. Screening potential $V_{\mathrm{scr}}(r)$ in Si plasmas under solar-interior conditions. The temperature and electron-density $\left(T / n_{\mathrm{e}}\right)$ values for the Si plasmas are taken from the standard solar model (Bahcall \& Ulrich 1988; Guenther et al. 1992): $156.90 \mathrm{eV} / 5.88 \times 10^{22} \mathrm{~cm}^{-3}$ (black longdashed-dotted line), $189.00 \mathrm{eV} / 8.0 \times 10^{22} \mathrm{~cm}^{-3}$ (black short-dasheddotted line), $205.12 \mathrm{eV} / 9.89 \times 10^{22} \mathrm{~cm}^{-3}$ (black long-dashed line), $227.53 \mathrm{eV} / 1.36 \times 10^{23} \mathrm{~cm}^{-3}$ (black short-dashed line), $244.94 \mathrm{eV} / 1.7$ $\times 10^{23} \mathrm{~cm}^{-3}$ (black dotted line), $275.80 \mathrm{eV} / 2.76 \times 10^{23} \mathrm{~cm}^{-3}$ (violet dashed-dashed-dotted line), $304.23 \mathrm{eV} / 4.06 \times 10^{23} \mathrm{~cm}^{-3}$ (turquoise dashed-dotted-dotted line), $334.40 \mathrm{eV} / 6.12 \times 10^{23} \mathrm{~cm}^{-3}$ (brown longdashed-dotted line), $373.18 \mathrm{eV} / 9.79 \times 10^{23} \mathrm{~cm}^{-3}$ (yellow short-dasheddotted line), $407.65 \mathrm{eV} / 1.43 \times 10^{24} \mathrm{~cm}^{-3}$ (blue long-dashed line), 438.70 $\mathrm{eV} / 1.96 \times 10^{24} \mathrm{~cm}^{-3}$ (green short-dashed line), $468.84 \mathrm{eV} / 2.57 \times$ $10^{24} \mathrm{~cm}^{-3}$ (red dotted line), and $496.42 \mathrm{eV} / 3.25 \times 10^{24} \mathrm{~cm}^{-3}$ (black solid line).

of the IPD is very important for dense plasmas. From the comparison shown in Fig. 1, we are confident that our model of plasma screening is valid for hot, dense plasmas.

Having validated our model by utilizing the IPD, we then used it to investigate the plasma screening potential for dense plasmas under solar-interior conditions. By using Si as an example, we show the screening potential produced by the plasma as a function of the distance from the center of the involved ion in Fig. 2. The electron temperature and density are taken from the standard solar model (Bahcall \& Ulrich 1988; Guenther et al. 1992). The highest temperature and density are $496.42 \mathrm{eV}$ and $3.25 \times 10^{24} \mathrm{~cm}^{-3}$, respectively, and they correspond to the plasma conditions at $0.3529 R_{\odot}$ (where $R_{\odot}$ is the solar radius), whereas the lowest values, $156.90 \mathrm{eV}$ and $5.88 \times 10^{22} \mathrm{~cm}^{-3}$, are the conditions at $0.7523 R_{\odot}$. We note that the electron temperature and density at the solar radiation and convection zone boundary, which is located at $0.7133 R_{\odot}$ (Bahcall et al. 1982), are determined to be $189.35 \mathrm{eV}$ and $8.08 \times 10^{22} \mathrm{~cm}^{-3}$.

Inspection of Fig. 2 reveals features of the additional potential exerted by the plasma environment on the involved $\mathrm{Si}$ ion. First, at any given temperature and density, the potential induced by the environment increases rapidly from a negative value at a distance very near the nucleus of an ion to a positive maximum and then gradually decreases with increasing distance from the ionic center. The negative potential that is very close to the nucleus means that the contributions from the exchange interaction exceed the Coulombic interaction, which is

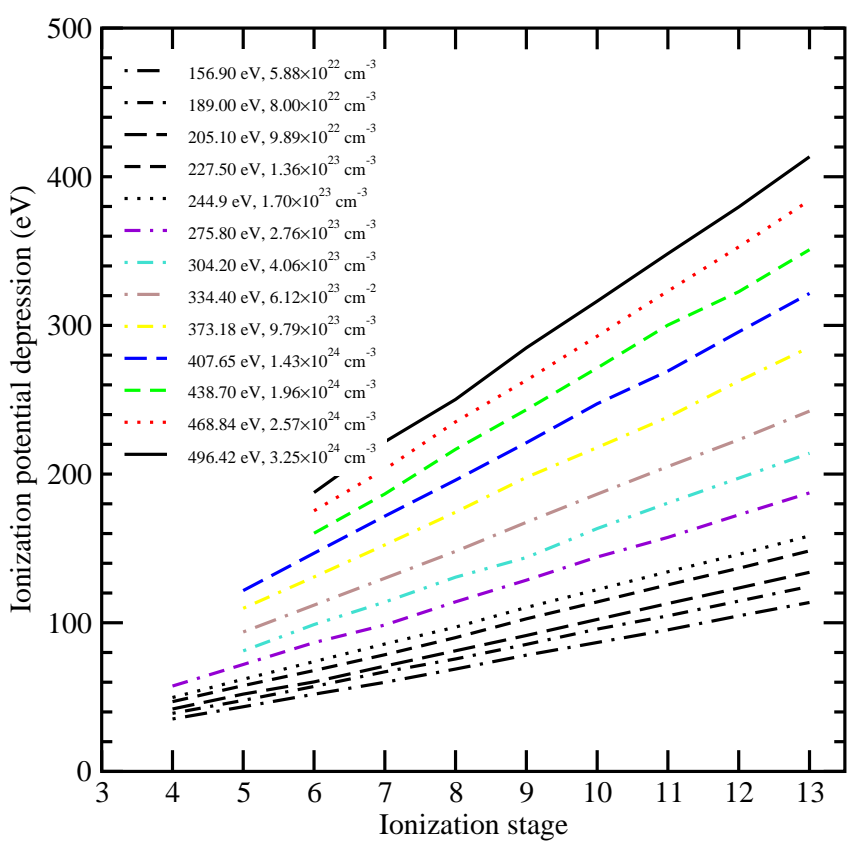

Fig. 3. Ionization-potential depressions of $\mathrm{Si}$ plasmas at the electron densities of $5.88 \times 10^{22} \mathrm{~cm}^{-3}$ (black long-dashed-dotted line), $8.0 \times$ $10^{22} \mathrm{~cm}^{-3}$ (black short-dashed-dotted line), $9.89 \times 10^{22} \mathrm{~cm}^{-3}$ (blacklongdashed line), $1.36 \times 10^{23} \mathrm{~cm}^{-3}$ (black short-dashed line), $1.7 \times 10^{23} \mathrm{~cm}^{-3}$ (black dotted line), $2.76 \times 10^{23} \mathrm{~cm}^{-3}$ (violet dashed-dashed-dotted line), $4.06 \times 10^{23} \mathrm{~cm}^{-3}$ (turquoise dashed-dotted-dotted line), $6.12 \times 10^{23} \mathrm{~cm}^{-3}$ (brown long-dashed-dotted line), $9.79 \times 10^{23} \mathrm{~cm}^{-3}$ (yello short-dasheddotted line), $1.43 \times 10^{24} \mathrm{~cm}^{-3}$ (blue long-dashed line), $1.96 \times 10^{24} \mathrm{~cm}^{-3}$ (green short-dashed line), $2.57 \times 10^{24} \mathrm{~cm}^{-3}$ (red dotted line), and $3.25 \times 10^{24} \mathrm{~cm}^{-3}$ (black solid line). They are taken from the standard solar model (Bahcall \& Ulrich 1988; Guenther et al. 1992).

completely an effect of quantum mechanics. Thus plasma screening results in an increase in the energies of bound states and a depression of the ionization threshold. Secondly, at lower electron densities, the induced potential decreases very slowly with $r$. The induced potential becomes larger and the decreasing trend becomes stronger with increasing electron density, which means stronger screening for a denser plasma. Finally, the induced potential does not increase linearly with electron density; the temperature of the plasma also has an effect on it. At the lowest density that we consider, $5.88 \times 10^{22} \mathrm{~cm}^{-3}$ $\left(T_{\mathrm{e}}=156.90 \mathrm{eV}\right)$, the peak value of the induced potential is 2.66 a.u. This peak value increases by factors of 1.11 and 3.20 at the densities of $4.06 \times 10^{23} \mathrm{~cm}^{-3}(304.23 \mathrm{eV})$ and $3.25 \times 10^{24} \mathrm{~cm}^{-3}$ (496.42 eV), respectively.

Figure 3 shows the calculated IPDs for Si plasmas under the same conditions as in Fig. 2. According to the calculations of ionization equilibrium determined by the DLAYZ code (Gao et al. 2013), the dominant ionization stages are predicted to be $\mathrm{Si}^{8+}-\mathrm{Si}^{13+}$ under the given plasma conditions. The DLAYZ code was developed to investigate the population kinetics and radiative properties of plasmas by solving the Saha ionization equilibrium equation or coupled rate equations. For dense plasmas, the plasma screening effect is included in the calculations of the basic atomic data. The first ionization potentials are $351.28 \mathrm{eV}, 401.38 \mathrm{eV}, 476.27 \mathrm{eV}, 523.42 \mathrm{eV}, 2437.66 \mathrm{eV}$, and $2673.18 \mathrm{eV}$ for isolated ions of $\mathrm{Si}^{8+}-\mathrm{Si}^{13+}$, respectively (Kramida et al. 2019). However, if these ions are immersed in a dense-plasma environment, the ionization potential is significantly decreased. For example, at the electron temperature 
and density of $496.42 \mathrm{eV}$ and $3.25 \times 10^{24} \mathrm{~cm}^{-3}$, the ionization potentials of $\mathrm{Si}^{8+}-\mathrm{Si}^{13+}$ are lowered to $250.22 \mathrm{eV}, 284.91 \mathrm{eV}$, $316.27 \mathrm{eV}, 348.32 \mathrm{eV}, 379.62 \mathrm{eV}$, and $413.43 \mathrm{eV}$ by the plasma environment. These are significant decreases in the ionization potentials, which will evidently modify the wave functions of the continuum states and thus affect photoionization and electronion collisional ionization processes. For a plasma at a given temperature and density, the IPDs increase nearly linearly with an increasing ionization stage, particularly at lower densities. Only when arriving at some critical plasma condition is such a linear dependence on the ionization stage slightly modified. For example, at the density of $4.06 \times 10^{23} \mathrm{~cm}^{-3}(304.23 \mathrm{eV})$, the IPD of $\mathrm{Si}^{9+}$ deviates slightly from a linear relation. This is because the bound $4 s$ orbital predicted at an electron density lower than $4.06 \times 10^{23} \mathrm{~cm}^{-3}$ begins to dissolve into continuum states at higher densities. The lines representing the IPDs become steeper with increasing density. At a density of $5.88 \times 10^{22} \mathrm{~cm}^{-3}(156.90 \mathrm{eV})$, the IPDs for $\mathrm{Si}^{6+}$ and $\mathrm{Si}^{13+}$ are $52.0 \mathrm{eV}$ and $113.6 \mathrm{eV}$, respectively. The corresponding values are $187.6 \mathrm{eV}$ and $413.4 \mathrm{eV}$ at $3.25 \times 10^{24} \mathrm{~cm}^{-3}(496.42 \mathrm{eV})$, which increase $135.6 \mathrm{eV}$ and $299.8 \mathrm{eV}$, respectively. The slopes of the IPD lines are thus 8.8 and 32.3 at the lowest and highest densities given, respectively.

In Fig. 4 we compare our predicted IPDs with those from the analytical models of Ecker \& Kröll (Ecker \& Kröll 1963), the ion sphere (Rozsnyai 1972), Stewart \& Pyatt (Stewart \& Pyatt 1966), and Debye \& Hückel (Debye \& Hückel 1923) at a few selected electron temperatures and densities: $156.90 \mathrm{eV}$ and $5.88 \times 10^{22} \mathrm{~cm}^{-3}, 227.53 \mathrm{eV}$ and $1.36 \times 10^{23} \mathrm{~cm}^{-3}, 407.65 \mathrm{eV}$ and $1.43 \times 10^{24} \mathrm{~cm}^{-3}$, and $496.42 \mathrm{eV}$ and $3.25 \times 10^{24} \mathrm{~cm}^{-3}$ We find that the Ecker-Kröll model (Ecker \& Kröll 1963) always predicts stronger screening and thus higher IPDs than our nonanalytical model, while the Debye-Hückel model (Debye \& Hückel 1923) always predicts weaker screening and lower IPDs than our model. The IPDs predicted by the ion sphere (Rozsnyai 1972) and Stewart-Pyatt (Stewart \& Pyatt 1966) models are very similar to our results for the lower ionization stages, but they deviate from our results for the increasing ionization stages; the higher the ionization stage, the larger the deviation. In addition, the results obtained from the ion-sphere (Rozsnyai 1972) and Stewart-Pyatt (Stewart \& Pyatt 1966) models agree reasonably well with each other for the lower ionization stages (less than nine), but the differences become larger for more highly charged states.

An accurate description of the plasma screening potential is of crucial importance for investigations of the atomic processes in dense plasmas (Liu et al. 2018). Recent experimental investigations on electron-impact ionization show that the cross-sections and rates are enhanced by dense-plasma effects and cannot be explained by currently available atomic collision theory (Vinko et al. 2015; van den Berg et al. 2018). Photoionization cross-sections are also increased, and thus the radiative opacity is increased as well for hot, dense plasmas (Bailey et al. 2015). The IPD is partially responsible for the enhancement of these continuum atomic processes.

\section{Summary and conclusions}

In this work, we propose a theoretical formalism for investigating the plasma screening potential of dense plasmas under solar-interior conditions. This screening is determined by the free-electron micro-space distribution obtained from ionization balance, which yields the average ionization in the plasma for an average-atom model. The contribution to the screening

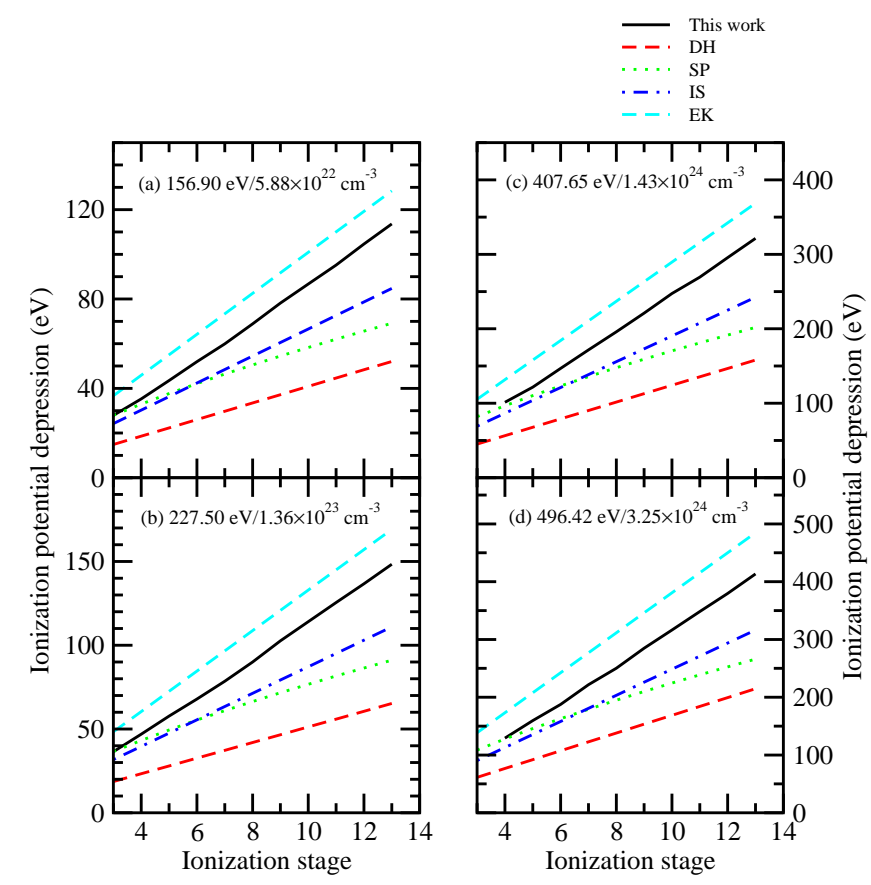

Fig. 4. Comparison of our theoretical ionization-potential depression with the analytical models of Debye-Hückel (DH) (Debye \& Hückel 1923), Stewart-Pyatt (SP) (Stewart \& Pyatt 1966), ion sphere (IS) (Rozsnyai 1972), and Ecker-Kröll (EK) (Ecker \& Kröll 1963) for a few selected plasma conditions of (a) $156.90 \mathrm{eV} / 5.88 \times 10^{22} \mathrm{~cm}^{-3},(b)$ $227.53 \mathrm{eV} / 1.36 \times 10^{23} \mathrm{~cm}^{-3}$, (c) $407.65 \mathrm{eV} / 1.43 \times 10^{24} \mathrm{~cm}^{-3}$, and $(d)$ $496.42 \mathrm{eV} / 3.25 \times 10^{24} \mathrm{~cm}^{-3}$.

from ions in the plasma is assumed to be trivial and hence is neglected. The ionization-potential depression is directly determined by the screening potential, and hence, we use it to check the validity of our model of the screening potential. We obtain the ionization-potential depression by computing the energy shifts of the involved electron orbitals with and without including plasma screening effects. A comparison with an experimental measurement of a solid-density Si plasma shows that our predicted ionization-potential depression agrees with the measured values, while the widely applied analytical ion-sphere (Rozsnyai 1972) and Stewart-Pyatt (Stewart \& Pyatt 1966) models usually underestimate the screening for highly charged states. We applied the proposed model to investigate the screening potential and ionization-potential depression of Si plasmas under solar-interior conditions from $0.7523 R_{\odot}$ to $0.3529 R_{\odot}$. The plasma screening potential provides the basis for further studies of atomic processes in dense plasma, including radiative transition, photoionization, and electron-impact excitation and ionization. We expect for it to find wideranging applications in astrophysics, plasma physics, atomic and molecular physics, high-energy-density physics, fusion science, and nonequilibrium plasmas produced by X-ray free-electron lasers.

Acknowledgements. This work was supported by Science Challenge Project No. TZ2018005, by the National Key R\&D Program of China under the grant No. 2017YFA0403202, and by the National Natural Science Foundation of China under Grant Nos. 11674394.

\section{References}

Ali, A., Naz, G. S., Shahzad, M. S., et al. 2018, High Energy Density Phys., 26, 48

Antia, H. M. 2009, Curr. Sci., 97, 911 
Bahcall, J. N., \& Ulrich, R. K. 1988, Rev. Mod. Phys., 60, 297

Bahcall, J. N., Huebner, W. F., Lubow, S. H., Parker, P. D., \& Ulrich, R. K. 1982, Rev. Mod. Phys., 54, 767

Bahcall, J. N., Serenelli, A. M., \& Pinsonneault, M. 2004, ApJ, 614, 464

Bailey, J. E., Nagayama, T., Loisel, G. P., et al. 2015, Nature, 517, 56

Basu, S., \& Antia, H. 2008, Phys. Rep., 457, 217

Blancard, C., Cossé, P., \& Faussurier, G. 2011, ApJ, 745, 10

Calisti, A., Ferri, S., \& Talin, B. 2015, J. Phys. B: At. Mol. Opt. Phys., 48, 224003

Christensen-Dalsgaard, J., \& Döppen, W. 1992, A\&ARv, 4, 267

Ciricosta, O., Vinko, S. M., Chung, H.-K., et al. 2012, Phys. Rev. Lett., 109, 065002

Ciricosta, O., Vinko, S. M., Barbrel, B., et al. 2016, Nat. Commun., 7, 11713

Crowley, B. 2014, High Energy Density Phys., 13, 84

Debye, P., \& Hückel, E. 1923, Phys. Z., 24, 185

Deprince, J., Bautista, M., Fritzsche, S., et al. 2019a, A\&A, 624, A74

Deprince, J., Bautista, M., Fritzsche, S., et al. 2019b, A\&A, 626, A83

Dharma-wardana, M. W. C., \& Taylor, R. 1981, J. Phys. C: Solid State Phys., 14, 629

Ecker, G., \& Kröll, W. 1963, Phys. Fluids, 6, 62

Fletcher, L. B., Kritcher, A. L., Pak, A., et al. 2014, Phys. Rev. Lett., 112, 145004

Gamow, G. 1928, Z. Phys., 51, 204

Gao, C., Zeng, J., \& Yuan, J. 2011, High Energy Density Phys., 7, 54

Gao, C., Zeng, J., Li, Y., Jin, F., \& Yuan, J. 2013, High Energy Density Phys., 9, 583

Gu, M. F. 2008, Can. J. Phys., 86, 675

Guenther, D., Demarque, P., Kim, Y., \& Pinsonneault, M. 1992, ApJ, 387, 373

Hansen, S. B., Faenov, A. Y., Pikuz, T. A., et al. 2005, Phys. Rev. E, 72, 036408

Hansen, S. B., Colgan, J., Faenov, A. Y., et al. 2014, Phys. Plasmas, 21, 031213

Hoarty, D. J., Allan, P., James, S. F., et al. 2013, Phys. Rev. Lett., 110, 265003

Hu, S. X. 2017, Phys. Rev. Lett., 119, 065001

Ichimaru, S. 1993, Rev. Mod. Phys., 65, 255

Iglesias, C. A. 2014, High Energy Density Phys., 12, 5

Iglesias, C. A., \& Rogers, F. J. 1991, ApJ, 371, 408

Janev, R., Zhang, S., \& Wang, J. 2016, Matter Radiat. Extremes, 1, 237

Jönsson, P., He, X., Fischer, C. F., \& Grant, I. 2007, Comput. Phys. Commun., 177,597

Kasim, M., Wark, J., \& Vinko, S. 2018, Sci. Rep., 8, 6276

Khrapak, S. A., Ivlev, A. V., Morfill, G. E., \& Zhdanov, S. K. 2003, Phys. Rev. Lett., 90, 225002
Kramida, A., Ralchenko, Y., Reader, J., \& NIST ASD Team. 2019, NIST Atomic Spectra Database (version 5.7.1), [Online]. Available: https://physics. nist.gov/asd [Wed Dec 04 2019]

Kraus, D., Bachmann, B., Barbrel, B., et al. 2018, Plasma Phys. Control. Fusion, 61, 014015

Li, Y., Wu, J., Hou, Y., \& Yuan, J. 2008, J. Phys. B: At. Mol. Opt. Phys., 41, 145002

Li, J., Zhang, S. B., Ye, B. J., Wang, J. G., \& Janev, R. K. 2017, Phys. Rev. A, 96, 032707

Lin, C., Röpke, G., Kraeft, W.-D., \& Reinholz, H. 2017, Phys. Rev. E, 96, 013202

Liu, P., Gao, C., Hou, Y., Zeng, J., \& Yuan, J. 2018, Commun. Phys., 1, 95

Porcherot, Q., Pain, J.-C., Gilleron, F., \& Blenski, T. 2011, High Energy Density Phys., 7, 234

Preston, T. R., Vinko, S. M., Ciricosta, O., et al. 2013, High Energy Density Phys., 9, 258

Qi, Y., Wang, J., \& Janev, R. 2009, Phys. Rev. A, 80, 063404

Rogers, F. J., \& Iglesias, C. A. 1994, Science, 263, 50

Röpke, G., Blaschke, D., Döppner, T., et al. 2019, Phys. Rev. E, 99, 033201

Rose, S. J. 1992, J. Phys. B: At. Mol. Opt. Phys., 25, 1667

Rosmej, F. B. 2018, J. Phys. B: At. Mol. Opt. Phys., 51, 09LT01

Rozsnyai, B. F. 1972, Phys. Rev. A, 5, 1137

Saiz, E. G., Gregori, G., Khattak, F., et al. 2008, Phys. Rev. lett., 101, 075003

Schnittman, J. D., Krolik, J. H., \& Noble, S. C. 2013, ApJ, 769, 156

Son, S.-K., Thiele, R., Jurek, Z., et al. 2014, Phy. Rev. X, 4, 031004

Stewart, J. C., \& Pyatt, Jr., K. D. 1966, ApJ, 144, 1203

Stransky, M. 2016, Phys. Plasmas, 23, 012708

van den Berg, Q., Fernandez-Tello, E., Burian, T., et al. 2018, Phys. Rev. Lett., 120,055002

Vinko, S., Ciricosta, O., Cho, B., et al. 2012, Nature, 482, 59

Vinko, S., Ciricosta, O., \& Wark, J. 2014, Nat. Commun., 5, 3533

Vinko, S., Ciricosta, O., Preston, T., et al. 2015, Nat. Commun., 6, 1

Yakovlev, D. G., \& Shalybkov, D. A. 1989, Sov. Sci. Rev. Ser. E: Astrophys. Space Phys., 7, 311

Yuan, J. 2002, Phys. Rev. E, 66, 047401

Zeng, J., \& Yuan, J. 2006, Phys. Rev. E, 74, 025401

Zeng, J., \& Yuan, J. 2007, Phys. Rev. E, 76, 026401

Zhang, S. B., Wang, J. G., \& Janev, R. 2010, Phys. Rev. Lett., 104, 023203 